\title{
Entropy-Constrained Gradient-Match Vector Quantization for Image Coding ${ }^{*}$
}

\author{
Shin-Chou Juan and Chen-Yi Lee \\ Dept. of Electronies Eng., National Chiao Tugn University \\ Hsinchu, Taiwan, ROC \\ Tel: +886-3-5731849; Email: cylee@cc.nctu.edu.tw
}

\begin{abstract}
:
Side-match VQ(SMVQ) is a well-known class of FSVQ used for low-bit rate image/video coding. It exploits the spatial correlation between the neighboring blocks to select several codewords that are very close to the encoding block from the master codebook. But if the block boundary is in the region edge area, the spatial correlations are not high and the SMVQ can't select proper codewords to encode blocks. In this paper, an Entropy-Constrained Gradient-Match VQ (ECGMVQ) is proposed. Instead of exploiting the spatial correlation, the ECGMVQ uses the gradient contiguity property to select the codewords. State function of ECGMVQ can select better codevectors than the SMVQ. In addition, the entropyconstrained rule is applied to the encoding process to reduce bit rate. Simulation results show that the improvement of ECGMVQ over the SMVQ is up to 4 5 dB at nearly the same bit rate. Further, the perceptual image quality is better than that of SMVQ, especially in the region edge area.
\end{abstract}

\section{INTRODUCTION}

Vector Quantization has been found to be an efficient technique for image compression. The images to be encoded are first processed to yield a set of vectors. Then a codebook is generated using sorne interative clustering algorithm, such as the generalized Lloyd clustering algorithm[1]. The input vectors are then individually quantized to the closest codewords in the codebook. Compression is achieved by using the indices of codewords for the purpose of transmission and storage.

However, an ordinary VQ exploits the high correlations between neighboring pixels, but ignores the high correlations between the neightoring blocks. To improve the compression ratio while maintaining high picture quality and avoid the excessive complexity, one way is to exploit memory for coding. Finite State VQ or FSVQ is a class of VQ with memory [2] Both encoder and decoder of an FSVQ have a finite state machine, which uses previously encoded vectors to decide current state and then selects one corresponding state codebook which is a subset of master codebook, to quantize input vector. Since the state codebook is smaller than the master codebook, FSVQ can achieve both highr compression ratio and lower computational cornplexity than ordinary VQ.

Side-match VQ(SMVQ) [3] is a well-known class of FSVQ's. SMVQ exploits the upper encoded block and left encoded block for the current input vector to predict the current encoding vector. SMVQ selects several codewords by measuring the side-match distortion function from the master codebook.
Codewords that produce less side-match distortion are selected as state codebook to encode input vector. In general, SMVQ is an efficient method for image coding and it makes the gray level transition across the boundaries of the vectors as smooth as possible. A lot of image coding algorithms are based on SMVQ and some modifications have been tried to achieve higher coding performance. In [4], a variable-rate Side-Match finitestate VQ with a block classifier (CSMVQ) is proposed. In CSMVQ, the size of the state codebook is changed according to the characteristics of the current vector which can be predicted by a block classifier. The improvement over SMVQ is up to $1.761 \mathrm{~dB}$. In [5], blocks are arranged into two main classes, namely edge blocks and nonedge blocks. Edge vectors are reclassified into 16 classes. Each class uses a different master codebook and uses SMVQ to encode input vectors. The improvement over ordinary SMVQ is up to $1.16 \mathrm{~dB}$ at nearly the same bit rate. In [6], three techniques: diagonal sampling, PCA, and rechecking are exploited in the proposed variable-rate SMVQ. The improvement over SMVQ is about 0.858-1.779 $\mathrm{dB}$.

Although a lot of modifications of SMVQ are proposed, the SMVQ distortion function have not been improved. In the complex area, correlations between the neighboring pixels are not high, then SMVQ selects codewords that are not close to input vector and results in a large quantization distortion. In this paper, a new Gradient-Match distortion function is proposed. Instead of using the spatial continuous property in SMVQ, the proposed GMVQ selects state codebook based on the gradient continuity property. By the proposed GMVQ distortion function, better state codebook can be selected to improve the coding quality and reduce output bit rate. The SMVQ distortion function in some image/video coding algorithm can be replaced directly by the proposed GMVQ distortion function to achieve better coding performance.

In addition, the optimal entropy-constrained rule is incorporated in the GMVQ to reduce the output index entropy. Although the coding quality degrades a little, better ratedistortion curve can be obtained with entropy-constrained rule. The improvement over ordinary SMQV is up to $4-5 \mathrm{~dB}$ at nearly the same bit rate.

\section{Coding Algorithm}

\subsection{Side-Match Vector Quantization}

A vector quantization is defined as a mapping from $k$ dimensional Euclidean space $R^{k}$ to a finite subset $C$ of $R^{k}$. We

\footnotetext{
- Work supported by the National Science Council of Taiwan, R.O.C. under Grant NSC-87-2215-E-009-035.
} 
call the finite set $C=\left\{c_{i} ; i=1, \ldots, N\right\}$ the codebook, where $c_{i}$ is a codeword and $\mathrm{N}$ is the codebook size.

FSVQ is a class of VQ with memory. The finite state machine of FSVQ uses previously encoded vectors to decide current state and then selects one corresponding state codebook, which is a subset of master codebook, to quantize input vector. Since the state codebook is smaller than the master codebook, FSVQ can achieve both higher compression ratio and lower computational complexity than ordinary VQ. Side-match vector quantization is one branch of FSVQ. Fig.l shows the relationship of image blocks in SMVQ. Let $x$ denote the current processing block, and $u$ and $l$ be the codewords of the upper and left neighboring blocks, respectively. SMVQ assumes that the correlations between the neighboring blocks are statistically high. The side-match distortion $d_{s m}$ between the vector $x$ and each codeword $c_{i}$ is defined as

$v d(y)$, the vertical side-match distortion:

$$
v d(y)=\sum_{j=1}^{m-1}\left(u_{m-h, j}-y_{0, j}\right)^{2}
$$

and $h d(y)$, the horizontal side-match distortion:

$$
h d(y)=\sum_{i=1}^{m-1}\left(l_{i, m-1}-y_{i, 0}\right)^{2}
$$

Thus the side-match distortion of a codeword $y$ is:

$$
d_{s m}(y)=h d(y)+v d(y)
$$

SMVQ sorts the codebook $C$ according to the side-match distortion $d_{s w}$ of codewords, and the first $N_{s}$ smallest-distortion codewords are selected as state codebook. For encoding, SMVQ picks the nearest codeword of $x$ from the state codebook, and employs the index of this nearest codeword in the state codebook to replace $x$.

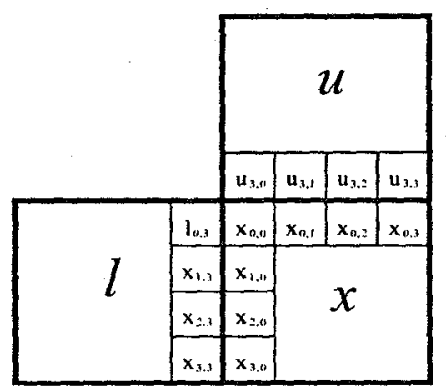

Fig. I: SMVQ uses both upper and left neighboring blocks to generate the state codebook.

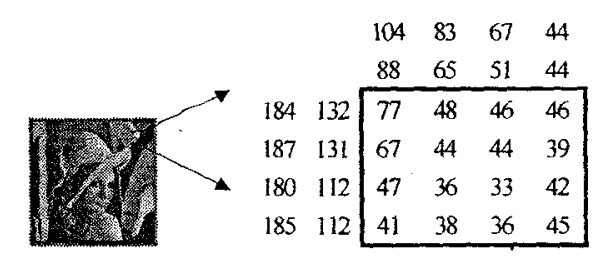

Fig. 2: Parts of $512 \times 512$ "Lena" image data.

\subsection{GMVQ}

When the image correlations are high, the SMVQ distortion function can select good state codebook to encode the input image. But in the complex area of an image, the SMVQ distortion is not acceptable. Fig 2 shows parts of the image data "Lena". It can be seen that the pixel differences between the encoding block $x$ and the neighboring blocks $u$ and $l$ are large and the distortion value calculated with SMVQ distortion function will become very larger. Thus the state codebook selected with SMVQ distortion function can't encode the image well.

A new distortion function, namely Gradient-Match VQ (GMVQ), is proposed here to improve the SMVQ distortion function. Instead of using the spatial continuity property, the GMVQ selects the state codebook by the gradient continuity property. In Fig. 2, it is shown that, although the spatial value of $x_{0,0}(=77)$ differs from that of neighboring pixel $u_{3,0}(=88)$ and $l_{0,3}(=132)$, the vertical gradient of $x_{0,0}(=88-77)$ is close to the vertical gradient of $u_{3,0}(=104-88)$ and the horizontal gradient of $x_{0,0}(=132-77)$ is also close to the vertical gradient of $u_{3,0}$ $(=184-132)$. Therefore, the new distortion function of GMVQ is defined as:

$v d^{\prime}(y)$, the vertical gradient-match distortion:

$\left.\left.d(y)=\sum_{j=0}^{m-1}\left(u_{n+2, j}-u_{n-L, j}\right)-\left(u_{m-1, j}-y_{0, j}\right)\right]^{2}+\sum_{j=1}^{m-1}\left(u_{n-1, j}-y_{0, j}\right)-\left(y_{0, i}-y_{1, j}\right)\right]^{2}$

and $h d^{\prime \prime}(y)$, the horizontal gradient-match distortion:

$h d^{\prime \prime}(y)=\sum_{i=1}^{m-1}\left[\left(l_{j, n-2}-l_{i, n-1}\right)-\left(l_{i, n-1}-y_{j, 0}\right)\right]^{2}+\sum_{i=1}^{m-1}\left[\left(l_{i, n-1}-y_{i, n}\right)-\left(y_{i, 0)}-y_{j, .}\right)\right]^{2}$

Thus, the gradient-match distortion of a codeword $y$ is defined as:

$$
\operatorname{gmd}(y)=h d^{\prime}(y)+v d^{\prime}(y)
$$

Because the region edge may be in different places, two items are needed in each distortion function. In the flat area, the GMVQ distortion behaves almost the same as the SMVQ distortion function. The difference of GMVQ and SMVQ is in the selection of state codebook, which is accomplished in designing state codebook process. Hence, although the GMVQ distortion function is more complex than SMVQ distortion function, no extra computation overhead is needed in the coding process.

\section{$2.3 \quad$ Entropy-Constrain GMVQ}

For both GMVQ and SMVQ, the codewords in the state codebook are sorted by the distortion measure, the codevectors produced smaller distortion are in the lower index parts of the state codebook. If the distortion measure can match the property of the input image, the output indices often lies in lower parts. Therefore, entropy coding, such as Huffman code, can be used to reduce the necessary bit rate of the indices. Shorter symbols are assigned to encode the lowir index, because their appearance probability is higher. Hence, the codewords in the state codebook have different cost in the transmitting of indices.

In general coding process, the nearest codevectors are always selected. Whether the improvement of nearest codewords over the other codewords is worth the cost in transmitting the channel symbol is not considered. In the encoding process, if we can measure the coding improvement and the transmission 
cost of codevector indices, a lot of bits can be saved with a little degradation of coding results, since the selected codevectors may be not the nearest.

In [7], Entropy-Constrained Vector Quantization (ECVQ) is proposed. The design problem is posed as the minimization of the Lagrangian $L=D+j R$, where $D$ is the expected distortion, $R$ is the rate (reproduction entropy), and $\lambda$ is the Lagrange multiplier whose value determines a particular rate-distortion tradeoff. It has been shown that ECVQ outperforms those obtained from a number of other variable-rate quantization schemes.

Here the Entropy-Constrained rule is applied to GMVQ, and the rate constrain causes a modification of the nearest neighboring rule to include an entropy cost based on the codeword probability $\mathrm{p}_{\mathrm{j}}$, where $p_{j}$ is the probability that $j$ th codeword in the state codebook is selected to encode the input image. The optimal entropy-constrained rule is:

$y_{j}$ is selected only if

$$
d\left(x, y_{j}\right)-\lambda \log p_{i}<=d\left(x, y_{k}\right)-\lambda \log p_{k}, \forall k
$$

By this criterion, the higher probability codewords will be more easily to be selected. And because their entropy codes are shorter, the resulting bit rate can be lowered with a little degradation of coding quality.

\section{Simulation Results}

Simulations have been performed for several $512 \times 512$ monochrome still images with 256 gray levels. The codebooks are generated by the Linde-Buzo-Gray algorithm from a training set of six different images. To evaluate coding performance, the PSNR between the original image and the encoded image has been calculated, where the PSNR is defined as

$$
\text { PSNR }=10 \log _{10} \frac{255^{2}}{M S E} d B
$$

Note that the mean square error for an $\mathrm{nXn}$ image is defined as

$$
\operatorname{MSE}=\left(\frac{1}{n}\right)^{2} \sum_{i=1}^{n} \sum_{i=1}^{n}\left(x_{i i}-\tilde{x}_{i j}\right)^{2}
$$

where $X_{i j}$ and $\tilde{X}_{i j}$ denote the original and encoded gray levels, respectively.

To compare the GMVQ distortion function with the SMVQ distortion function, we encode the Lena image with fixed master codebook size $=512$ and change the state codebook size. The output indices are compressed with Huffman code to reduce bit rate. Fig. 3 shows the PSNR values and bit rates at state codebook size $=8,16,32,64,128$ and 256. At the same state codebook size, the PSNR of GMVQ are always higher than that of SMVQ. This is because the GMVQ distortion function is more close to the image property than SMVC The output bit rates of GMVQ are lower than that of SMVQ, because the state codebooks sorted by the GMVQ are better than that of SMVQ. At the same bit rate, the PSNR of GMVQ are $1.5 \mathrm{~dB}$ higher than that of SMVQ. The improvements of GMVQ over SMVQ in other images are similar.

To compare the vision effect of GMVQ and SMVQ, an extreme case (state codebook size $=8$ ) is simulated. Fig. $4(a)$ is the original image of Lena. Fig. $4(\mathrm{~b})$ and Fig. $4(\mathrm{c})$ are the decoded images of SMVQ and GMVQ respectively. In Fig. 4(b), the white parts of test image Lena's right eye disappears. This is because the SMVQ distortion function can't select proper state codebook at the complex area. And in Fig. 4(c), although the state codebook size is small, GMVQ can encode the white parts of the Lena's right eye well. The difference of Fig. 4(b) and Fig. 4(c) is shown in Fig. 4(d). Most of the improvements of GMVQ over SMVQ are in the region edge area. For the flat area, the coding results remain the same.

To incorporate the Entropy Constrain Rules into GMVQ algorithm, 5 images are encoded by the proposed GMVQ to get the probability distributions of the state codebook indices. And this probability distribution is used in the entropy-constrained rule during encoding process. Fig. 5 shows the performance of ECGMVQ at different Lagrange multiplier $\lambda$ value and original SMVQ. As $\lambda$ increases, the bit rate will decrease a lot with a little PSNR degradation. At $\lambda=300$, the proposed ECGMVQ can achieve $30.05 \mathrm{~dB}$ at $0.18 \mathrm{bit} / \mathrm{pixel}$ and the improvement over SMVQ is up to $5 \mathrm{~dB}$ at similar bit rate. The decoded image of Lena with ECGMVQ at $\lambda=300$ and state codebook size $=256$ is shown is Fig. 6.

\section{CONCLUSION}

In this paper, a new Gradient-Match VQ distortion function is proposed to improve the selection of state codebook in FSVQ design processing. And simulation results show that at the same state codebook size the GMVQ can achieve better coding results, especially in the region edge area, and the bit rate is lower. These improvements don't need any extra computation overhead than SMVQ in the coding process. The GMVQ distortion function can be used to replace the SMVQ distortion function of other coding algorithms to improve the selection of state codebook.

Furthermore, by incorporating the Entropy-Constrained rule, the proposed ECGMVQ can obtain up to 4 $5 \mathrm{~dB}$ over SMVQ at nearly the same bit rate. Simulation result does show that our proposal is very promising for very low bit rate image coding.

\section{REFERENCES}

[1] Y. Linde, A. Buzo, and R. M. Gray, "An algorithm for Vector Quantizer design," IEEE Trans. Com., vol. COM-28, pp. 84-95, Jan. 1980.

[2] J. Foster, R.M. Gray and M. O. Dunham, "Finite state vector quantizer for wave coding," IEEE Trans. Inform Theon. vol. IT-31, pp. 348-355, May 1985.

[3] T. Kim, "Side match and overlap match vector quantization for images," IEEE Trans. Image Processing., vol. 1, no. 2, pp. 170 185, Apr. 1992.

[4] R. F. Chang and W. T. Chen, "Image coding using variable-rate side-match finite-state vector quantization," IEEE Trans. Image Processing., vol. 2 pp. 104-108, Jan. 1993

[5] R. F. Chang and W. M. Chen, "Adaptive Edge-Based Side-Match Finite- state classified vector quantization with quadtree map," IEEE Trans. Image Processing.vol.5 No. 2 pp. 378-383, Feb. 1996.

[6] T. S. Chen and C. C. Chang, "An new image Coding Algorithm Using variable-rate side-match finite-state vector quantization," IEEE Trans. Image Processing., vol. 6, 100. 8, pp. 1185-1187, Aug. 1997.

17] P. A. Chou, T. Lookabaugh, and RM Gray "Entropyconstrained vector quantization,"IEEE Trans. ASSP., vol. 37. pp. $31-42,1989$. 


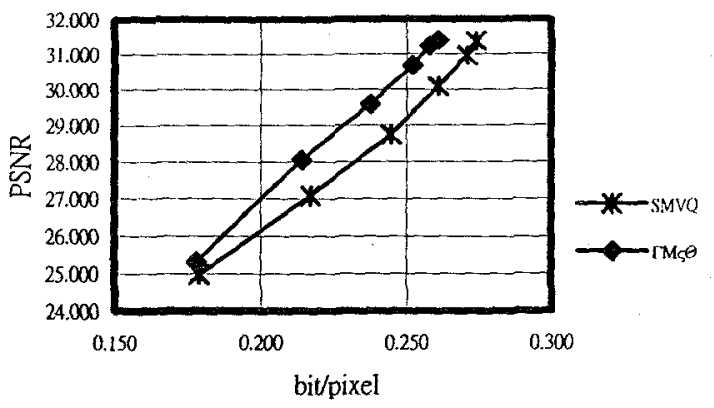

Fig. 3: The Comparisons of GMVQ and SMVQ for test image Lena. The master codebook size $=512$, and the state codebook sizes are 8, 16, 32, 64, 128 and 256. The indices are compressed with Huffman code.

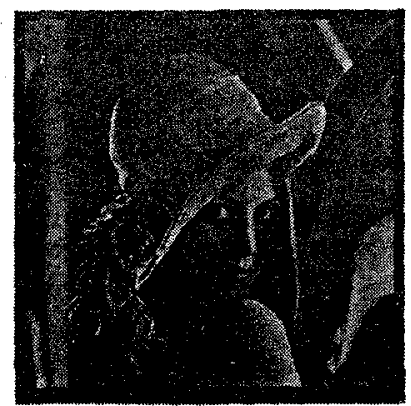

(a)

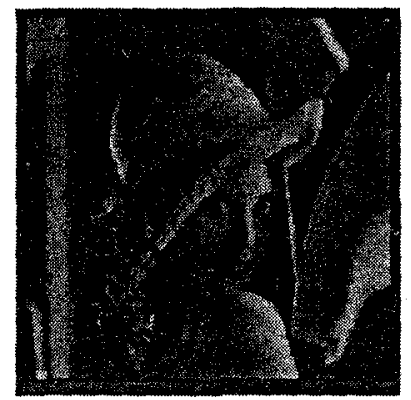

(c)

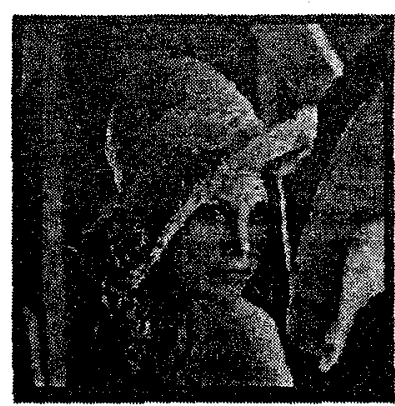

(b)

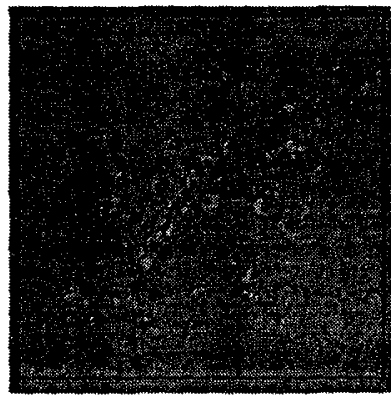

(d)
Fig. 4: An extreme case to compare the visual coding results of GMVQ and SMVQ. (master codebook size $=512$, state codebook size=8). (a) is the original $512 \times 512$ Lena image, (b) is the decoded image by $S M V Q$, (c) is the decoded image by GMVQ and (d) is the difference of (b) and (c).

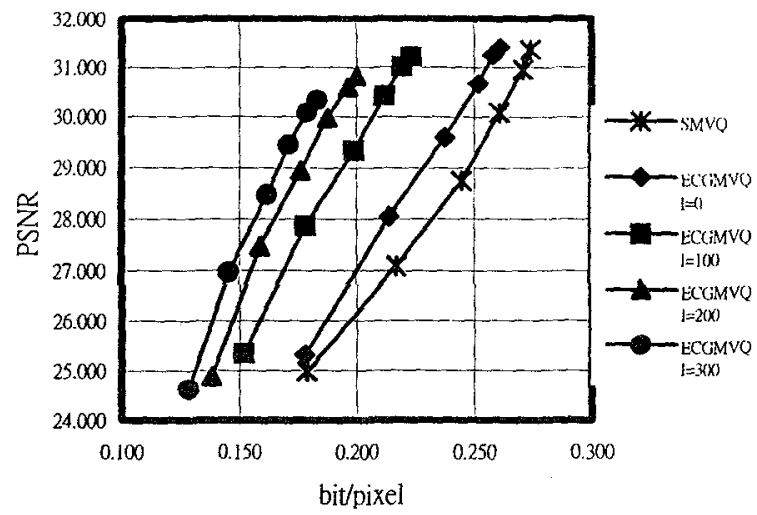

Fig. 5: The Comparisons of ECGMVQ at different Lagrange multiplier $\lambda$ value and $S M V Q$ for test image Lena. The master codebook size $=512$, and the state codebook sizes are 8, 16, 32, 64,128 and $256, \lambda$ varies from $0,100,200$, and 300 . The indices are compressed with Huffman code.

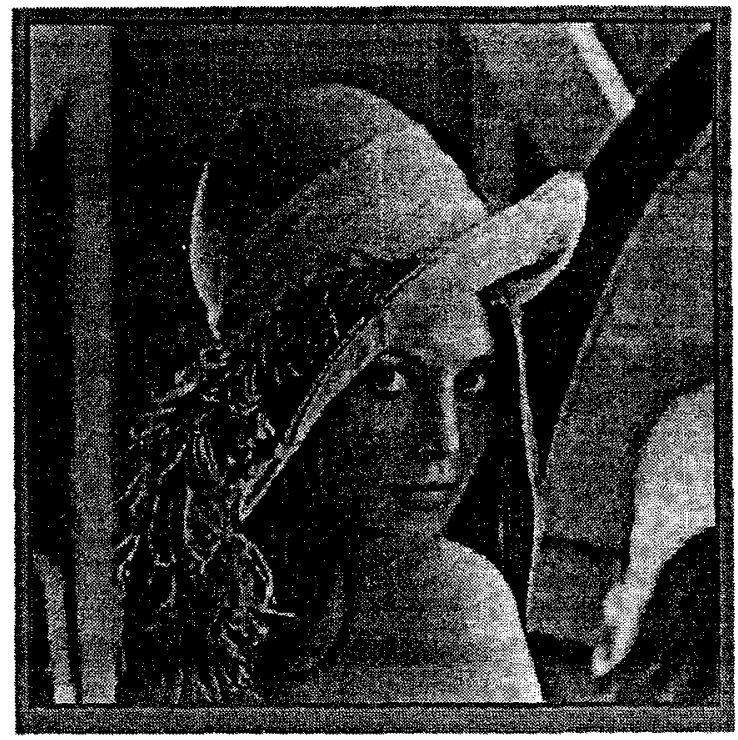

Fig. 6: The encoded image by the proposed ECGMVQ (master codebook size $=512$, state codebook size $=256, \lambda=300$ ). The PSNR is $30.34 \mathrm{~dB}$ and the bit rate is 0.183 bit/pixel. 